\title{
PERFORMANCE OF FADING MULTI-USER DIVERSITY FOR UNDERLAY COGNITIVE NETWORKS ${ }^{\dagger}$
}

\author{
Fahd Ahmed Khan ${ }^{\star}$,Mérouane Debbah ${ }^{\bullet}$,Kamel Tourki* and Mohamed-Slim Alouini ${ }^{\star}$ \\ ${ }^{\star}$ CEMSE Division, KAUST, Thuwal, Makkah Province, Saudi Arabia. Email: \{fahd.khan,slim.alouini\} @ kaust.edu.sa \\ -Alcatel-Lucent Chair - SUPELEC, Gif-sur-Yvette, France. Email: \{merouane.debbah\}@ supelec.fr \\ *ECEN Program, Texas A\&M Univ. at Qatar, Education City, Doha, Qatar. Email: \{kamel.tourki\}@qatar.tamu.edu
}

\begin{abstract}
Having multiple secondary users (SUs) can be exploited to achieve multiuser diversity and improve the throughput of the underlay secondary network. In the cognitive setting, satisfying the interference constraint is essential, and thus, a scheduling scheme is considered where some SUs are preselected based on the low interference power. From this subset, the SU that yields the highest throughput is selected for transmission. This scheduling scheme helps to lower the interference power while giving good throughput. For an independent but not identically distributed Nakagami-m fading channel, we obtain exact closed-form expressions of the capacity of this scheduling scheme. Furthermore, the scheduling time of SUs is characterized and closed-form expressions for the mean time after which a SU is selected for transmission are obtained. Numerical simulations are performed to corroborate the derived analytical results. Our results show that at low interference threshold, increasing transmit power of the SUs is not beneficial and results in reduced capacity. Furthermore, the channel idle time (i.e. time that no user is utilizing the channel) reduces with increasing the number of SUs.
\end{abstract}

Index Terms - Cognitive Radio, Multi-user Diversity, Ergodic Capacity, Fading Channels.

\section{INTRODUCTION}

Cognitive radio has been recently proposed as a solution to improve the utilization of the spectrum by spectrum sharing where the cognitive network transmits using the spectrum already allocated to some primary network [1,2]. One of the approaches for spectrum sharing is the underlay approach in which the cognitive/secondary network can transmit concurrently with the primary network only if it does not cause harmful interference to the primary network.

Satisfying the interference constraint imposed by the primary network results in performance degradation of the sec-

\footnotetext{
${ }^{\dagger}$ This work was supported in part by King Abdullah University of Science and Technology (KAUST).
}

ondary system. This performance degradation can be compensated by exploiting the best channels which become available when there are more users or mobility [3]. By selecting the user that has the best channel gives rise to a multiuser diversity effect which results in improved quality-ofservice $(\mathrm{QoS})$ and an increase in the overall capacity of the network [4]. Further diversity gains can be achieved by using opportunistic antennas [5]. However, a drawback of this scheme is that the users with the bad channels get selected less often, thus, these users suffer large delays in communication.

In a spectrum sharing setting, multi-user diversity can be exploited but it is different from the traditional multi-user case because of the interference power constraints imposed on the secondary user (SU). Thus, in this case, the selected SU needs to have jointly a good transmission link and a poor interference link. This idea can be achieved in various ways such as by using a precoder [6] or through the use of antennas [7]. However, in this paper, we exploit the multiple users in the secondary network. Due to the random fading, it is highly probable that some SU transmitter (SU-Tx) has the interference link (SU-Tx-to-primary user receiver (PU-Rx) link) in a deep fade and a very good SU-Tx-to-SU-Rx link. Thus, the SUs can be scheduled when the interference link is on a fade.

The effect of multi-user diversity in an underlay network with independent and identically distributed (i.i.d) Rayleigh fading was initially studied in [8]. It was shown in [8] that at sufficiently high transmit power, the selection of the user is more influenced by the interference link and the multi-user diversity gain in terms of capacity scales similar to the scaling law of the non-spectrum sharing system. The ergodic capacity of the multi-user underlay system was derived for a more generic hyper-Nakagami-m fading channel in [9]. The outage capacity as well as the effective capacity for an opportunistic cognitive broadcast channel having i.i.d Rayleigh fading was analyzed in [10]. Multi-user interference diversity which takes into account the interference from the primary network was studied in [11]. In [12], a new hybrid scheduling scheme was proposed which selects SUs for transmission in two steps; 1) First a subset of SUs is selected that have the interference link in deep fade and 2) then the SUs with the best transmis- 
sion links are selected from the pre-selected subset. The authors in [12] also gave bounds on the throughput of the hybrid scheduling scheme for a Rayleigh fading channel.

In this paper, we consider a variant of the hybrid scheduling scheme considered in [12], where only a single SU is chosen for transmission. Considering an independent but not identically distributed (i.n.i.d) Nakagami-m fading channel, we obtain closed form expression of the capacity of the scheduling scheme. In the hybrid scheduling scheme, it is possible that sometimes the initial subset is empty and thus no SU is selected for transmission. Thus, utilizing the renewal concept of a cycle, expressions for the mean time after which a SU is scheduled are derived. These expressions are useful in analyzing the feasibility of the network and adjusting network parameters. For example, the SUs in a network are usually equipped with finite buffers which might overflow if the SUs are not scheduled properly. Thus, the parameters of the network can be adjusted based on the mean scheduling time of the SU and the buffer lengths of each SU to avoid buffer overflow. Furthermore, numerical simulations are performed to corroborate the derived analytical results. Our results also show that at low interference thresholds, increasing transmit power of the SUs is not beneficial and results in reduced capacity. In addition, as the number of SUs increase the channel idle time reduces implying that for a large number SUs, there is always some $\mathrm{SU}$ which will be able to transmit.

\section{SYSTEM MODEL}

Assume that a secondary network is sharing the spectrum with a primary network. The secondary network consists of $L$ transmitters and a receiver. There is a single primary user receiver (PU-Rx) in the surroundings of the SU-Tx. Let the channel power gain between SU-Tx and $l$-th SU-Rx be denoted by $h_{l}$, and the channel power gain between $l$-th SUTx and PU-Rx is denoted by $g_{l}$. The channel is assumed to be i.n.i.d with Nakagami-m fading. Therefore, $h_{l}$ and $g_{l}$ are Gamma distributed. For a Gamma random variable X with parameters $m_{a}$ and $\Omega_{a}$, the probability distribution function (PDF) is given as $f_{X}(x)=\frac{1}{\Gamma\left(m_{h}\right)}\left(\frac{m_{a}}{\Omega_{a}}\right)^{m_{a}} x^{m_{a}-1} e^{-x \frac{m_{a}}{\Omega_{a}}}$ and the cumulative distribution function (CDF) is given as $F_{X}(x)=$ $\frac{\gamma\left(m_{a}, x \frac{m_{a}}{\Omega_{a}}\right)}{\Gamma\left(m_{a}\right)}=1-e^{-\frac{m_{a}}{\Omega_{a}}} \sum_{k=0}^{m_{a}-1} \frac{x^{k}}{k !}\left(\frac{m_{a}}{\Omega_{a}}\right)^{k}$ where, the second equality assumes that $m_{a}$ is an integer and $\gamma(\cdot, \cdot)$ denotes the lower incomplete gamma function [13, Eq. (8.350.1)]. The CDF and PDF of $g_{l}$ is obtained by replacing $X=g_{l}$ and $a=g_{l}$. Similarly, the CDF and PDF of $h_{l}$ is obtained by replacing $X=h_{l}$ and $a=h_{l}$. The noise at the nodes in the network is assumed to be zero mean complex Gaussian with variance $N_{0}$.

Selection Criteria: Let the maximum allowed interference power at the primary user be $Q$, then $P_{l} g_{l}<Q$, where $P_{l}$ is the transmit power of the $l$-th SU-Tx. For simplicity, assume that the maximum transmit power allowed at each SU-Tx is $P$ and also that the selected SU-Tx always transmits with power

\section{$P$. The SU-Tx for transmission is selected in two steps.}

1. First a subset of SU-Tx is selected that satisfy the interference power constraint i.e.

$$
T_{j}^{v}=\left\{l: g_{l}<\frac{Q}{P}, \forall l\right\},
$$

where $T_{j}^{v}$ denotes the $j$-th subset and is of size $v+1$ and contains indices of the SU-Tx whose interference link value is below $\frac{Q}{P}$ i.e. $T_{j}^{v} \in \mathcal{P}(U)$, where $U=$ $\left\{S U_{1}, S U_{2}, . . S U_{L}\right\}$ is the set of all available SU-Tx and $\mathcal{P}(\cdot)$ denotes the power set of its argument.

2. In the second step the SU-Tx with the best SU forward link is selected. Let, $T_{j}^{v}(k)$ denote the $k$-th element of set $T_{j}^{v}$, then the index of SU-Tx can be obtained as

$$
\iota=\arg \max _{k}\left\{h_{T_{j}^{v}(k)}\right\} \text {. }
$$

This procedure to select a SU-Tx is a special case of the scheduling scheme considered in [12].

\section{ERGODIC CAPACITY}

The ergodic capacity of this scheduling scheme can be obtained as

$$
C=\sum_{j} \operatorname{Pr}\left\{T_{j}^{v}\right\} C_{j},
$$

where $C_{j}$ is the ergodic capacity given that the selected subset is $T_{j}^{v}$ and $\operatorname{Pr}\left\{T_{j}^{v}\right\}$ (probability of selecting subset $T_{j}^{v}$ ) is

$$
\operatorname{Pr}\left\{T_{j}^{v}\right\}=\prod_{i_{1}=1, i_{1} \in T_{j}^{v}}^{L} F_{g_{i_{1}}}\left(\frac{Q}{P}\right) \prod_{i_{1}=1, i_{1} \notin T_{j}^{v}}^{L}\left(1-F_{g_{i_{1}}}\left(\frac{Q}{P}\right)\right)
$$

where $F_{g_{k}}\left(\frac{Q}{P}\right)=\operatorname{Pr}\left\{g_{k}<\frac{Q}{P}\right\}=\operatorname{Pr}\left\{k \in T_{j}^{v}\right\}$. For subset $T_{j}^{v}$, the end-to-end-SNR for the scheduling scheme is $\gamma_{j}=\frac{P}{N_{0}} \max _{i}\left\{h_{T_{j}^{v}(i)}\right\}$. Using [14, Eq. (9)] the CDF of $\gamma_{j}$, is

$$
F_{\gamma_{j}}(x)=1+\sum_{G_{v+1}} \bar{\kappa}_{n, p} e^{-x \bar{B}_{n}} x^{\bar{A}_{n, p}}
$$

where $\bar{\kappa}_{n, p}=\prod_{q=1}^{v+1}(-1)^{n_{q}}\left(\frac{m_{X_{j}^{v}(q)} N_{0}}{\Omega_{X_{j}^{v}(q)}{ }^{P}}\right)^{p_{q} n_{q}} \frac{m_{X_{j}^{v}(q)}{ }^{n_{q}-1}}{\left(p_{q} !\right)^{n} q}, \bar{B}_{n}=$ $\sum_{q=1}^{v+1} n_{q} \frac{m_{X_{j}^{v}(q)} N_{0}}{\Omega_{X_{j}^{v}(q)}\left(A^{P}\right.}, \bar{A}_{n, p}=\sum_{q=1}^{v+1} p_{q} n_{q}, \sum_{G_{v+1}}$ is a short hand no-

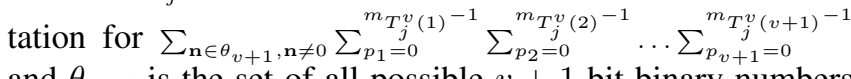
and $\theta_{v+1}$ is the set of all possible $v+1$ bit binary numbers and $n_{q}$ is the $q$-th bit of the binary number $\mathbf{n} \in \theta_{v+1}$. The ergodic capacity for the subset is $T_{j}^{v}$ is obtained using [15]

$$
C_{j}=\vartheta \int_{0}^{\infty} \frac{1-F_{\gamma_{j}}(x)}{1+x} d x=-\vartheta \sum_{G_{v+1}} \bar{\kappa}_{n, p} \int_{0}^{\infty} \frac{e^{-x \bar{B}_{n}} x^{\bar{A}_{n, p}}}{1+x} d x
$$

where $\vartheta=\frac{\log _{2}(e)}{B}$ and $B$ denotes the bandwidth. Solving the integral using [13, Eq. (3.383.10)] one gets

$$
C_{j}=-\frac{\log _{2}(e)}{B} \sum_{G_{v+1}} \bar{\kappa}_{n, p} e^{\bar{B}_{n}} \Gamma\left(\bar{A}_{n, p}+1\right) \Gamma\left(-\bar{A}_{n, p}, \bar{B}_{n}\right) .
$$

The capacity of the multiuser scheduling scheme can be obtained by substituting (7) in (3). 


$$
\begin{aligned}
& \mathcal{I}_{u}=\operatorname{Pr}\{u-\text { th SU-Tx is selected for transmission }\}=\mathcal{I}_{1}+\mathcal{I}_{2}= \\
& \operatorname{Pr}\left\{\text { Only } u \in T_{j}^{v}\right\}+\operatorname{Pr}\left\{u \text { and additional users are } \in T_{j}^{v}\right\} \operatorname{Pr}\left\{h_{u}>h_{T_{j}^{v}(k) \mid T_{j}^{v}(k) \neq u} ; \forall k\right\} ; \forall T_{j}^{v}
\end{aligned}
$$

\section{ANALYSIS OF THE USER SCHEDULING TIME}

\subsection{Probability that $u$-th SU is Selected:}

The probability that the $u$-th SU-Tx is selected for transmission can be expressed as (8), where $\mathcal{I}_{2}=\operatorname{Pr}\left\{u \in T_{j}\right\} \chi_{1}$,

$$
\mathcal{I}_{1}=\operatorname{Pr}\left\{\text { Only } u \in T_{j}^{v}\right\}=F_{g_{u}}\left(\frac{Q}{P}\right) \prod_{i_{1}=1, i_{1} \neq u}^{L}\left(1-F_{g_{i_{1}}}\left(\frac{Q}{P}\right)\right),
$$

and $\chi_{1}=\operatorname{Pr}\left\{T_{j}^{v}\right.$ has additional users $\} \operatorname{Pr}\left\{h_{u}>h_{T_{j}^{v}(k) \mid T_{j}^{v}(k) \neq u} ; \forall k\right\}$.

For a certain set $T_{j}^{v}$ having $v+1$ elements, conditioned on the fact that $u \in T_{j}^{v}$, then $T_{j}^{v}$ can be expressed as $T_{j}^{v}=$ $\left\{u, X_{j}^{v}\right\} . X_{j}^{v}$ is a set of indices of the SU-Tx in $T_{j}^{v}$ excluding $u$. Let $X_{j}^{v}(k)$ denote the $k$-th element of set $X_{j}^{v}$. As there are $L$ SU-Tx, so the number of possible elements in set $X_{j}^{v}$ can vary from 1 to $L-1$, therefore $1 \leq v \leq L-1$. Furthermore, for a fixed $v, X_{j}^{v}$ can have any of the $\left(\begin{array}{c}L-1 \\ v\end{array}\right)$ possible combinations of elements of set $E=\{1, . . u-1, u+1, . . L\}$, which is a set of indices denoting each SU-Tx excluding the $u$-th SU-Tx. Therefore, for a fixed $v, \chi_{1}$ is given by

$$
\chi_{1}=\sum_{v=1}^{L-1} \sum_{j=1}^{\left(\begin{array}{c}
L-1 \\
v
\end{array}\right)} \operatorname{Pr}\left\{X_{j}^{v}\right\} \operatorname{Pr}\left\{h_{u}>h_{X_{j}^{v}(i)} ; \forall i\right\} .
$$

Conditioned on $h_{u}, h_{X_{j}^{v}(k)}$ are independent, thus

$$
\operatorname{Pr}\left\{h_{u}>h_{X_{j}^{v}(i)} \mid h_{u} ; \forall i\right\}=\prod_{i=1}^{v} F_{h_{X_{j}^{v}(i)}}\left(h_{u}\right) .
$$

By averaging (11) using the PDF of $h_{u}$, yields

$$
\operatorname{Pr}\left\{h_{u}>h_{X_{j}^{v}(i)} ; \forall i\right\}=\int_{0}^{\infty} \prod_{i=1}^{v} F_{h_{X_{j}^{v}(i)}}\left(h_{u}\right) f_{h_{u}}\left(h_{u}\right) d h_{u} .
$$

Substituting $f_{h_{u}}(\cdot)$ and $F_{h_{X_{j}^{v}(i)}}(\cdot)$ into (12) and using [14, eq. (9)], (12) can be expressed as

$$
\begin{aligned}
& \operatorname{Pr}\left\{h_{u}>h_{X_{j}^{v}(i)} ; \forall i\right\}= \\
& \frac{1}{\Gamma\left(m_{h_{u}}\right)}\left(\frac{m_{h_{u}}}{\Omega_{h_{u}}}\right)^{m_{h_{u}}} \sum_{G_{v}} \kappa_{n, p} \int_{0}^{\infty} e^{-x B_{n}-x \frac{m_{h_{u}}}{\Omega_{h_{u}}}} x^{A_{n, p}+m_{h_{u}}-1} d x,
\end{aligned}
$$

where $\kappa_{n, p}=\prod_{q=1}^{v}(-1)^{n_{q}}\left(\frac{m_{X_{j}^{v}(q)}}{\Omega_{X_{j}^{v}(q)}}\right)^{p_{q} n_{q}} \frac{m_{X_{j}^{v}(q)}{ }^{n_{q}-1}}{\left(p_{q} !\right)^{n_{q}}}, A_{n, p}=$ $\sum_{q=1}^{v} p_{q} n_{q}, B_{n}=\sum_{q=1}^{v} n_{q} \frac{m_{X_{j}^{v}(q)}}{\Omega_{X_{j}^{v}(q)}^{v}}$ and $\sum_{G_{v}}$ is a short hand notation for $\sum_{\mathbf{n} \in \theta_{v}} \sum_{p_{1}=0}^{m_{X_{j}^{v}(1)}{ }^{-1}} \sum_{p_{2}=0}^{m_{X_{j}^{v}(2)}{ }^{-1}} \ldots \sum_{p_{v}=0}^{m_{X_{j}^{v}(v)}}{ }^{-1}$. Solving the integral in (13), yields

$$
\begin{aligned}
& \operatorname{Pr}\left\{h_{u}>h_{X_{j}^{v}(i)} ; \forall i\right\}= \\
& \sum_{G_{v}}\left(\frac{m_{h_{u}}}{\Omega_{h_{u}}}\right)^{m_{h_{u}}} \kappa_{n, p} \frac{\Gamma\left(A_{n, p}+m_{h_{u}}\right)}{\Gamma\left(m_{h_{u}}\right)}\left(B_{n}+\frac{m_{h_{u}}}{\Omega_{h_{u}}}\right)^{-\left(A_{n, p}+m_{h_{u}}\right)} .
\end{aligned}
$$

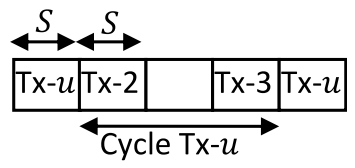

Fig. 1. Transmission Cycle.

Substituting (14) into (10) gives

$$
\begin{aligned}
\chi_{1}=\sum_{v=1}^{L-1} & \sum_{j=1}^{\left(\begin{array}{l}
L-1 \\
v
\end{array}\right.} \sum_{G_{v}} \operatorname{Pr}\left\{X_{j}^{v}\right\}\left(\frac{m_{h_{u}}}{\Omega_{h_{u}}}\right)^{m_{h_{u}}} \kappa_{n, p} \\
& \times \frac{\Gamma\left(A_{n, p}+m_{h_{u}}\right)}{\Gamma\left(m_{h_{u}}\right)}\left(B_{n}+\frac{m_{h_{u}}}{\Omega_{h_{u}}}\right)^{-\left(A_{n, p}+m_{h_{u}}\right)},
\end{aligned}
$$

where $\operatorname{Pr}\left\{X_{j}^{v}\right\}$ is given by

$$
\operatorname{Pr}\left\{X_{j}^{v}\right\}=\prod_{i_{1}=1, i_{1} \in X_{j}^{v}}^{L} F_{g_{i_{1}}}\left(\frac{Q}{P}\right) \prod_{i_{1}=1, i_{1} \notin X_{j}^{v}}^{L}\left(1-F_{g_{i_{1}}}\left(\frac{Q}{P}\right)\right) .
$$

Substituting, $\mathcal{I}_{2}=\chi_{1} F_{g_{u}}\left(\frac{Q}{P}\right)$ in (8) yields (17). (17) gives the probability that the $u$-th SU-Tx is selected for transmission The probability that no SU-Tx is selected is denoted by $\mathcal{I}_{0}$ and can be obtained as

$$
\mathcal{I}_{0}=1-\sum_{u=1}^{L} \mathcal{I}_{u}
$$

\subsection{Time Scheduling of Users}

Using the renewal concept of cycle, we find the average time after which $u$-th SU-Tx is selected for transmission [16]. Assume that whenever a SU-Tx is selected it transmits for $S$ seconds. A cycle is defined as the interval between two successive instances when a given SU-Tx is selected for transmission.

Fig. 1 shows an example of a cycle for the $u$-th SU-Tx. It can be observed in Fig. 1 that once a SU-Tx is selected, it transmits for $S$ seconds. The empty interval implies that no SU-Tx is chosen to transmit in that interval. The inter-arrival time for SU-Tx $u$, is denoted by $A_{u}$, and can be expressed as

$$
A_{u}=\sum_{l=0, l \neq u}^{L} \sum_{i=1}^{N_{l}^{u}} S=S \sum_{l=0, l \neq u}^{L} N_{l}^{u}
$$

where $N_{l}^{u}$ is the number of times that the $l$-th SU-Tx is chosen for transmission during the cycle corresponding to the $u$-th SU-Tx. Note that $N_{l}^{u}$ is a random variable. For the mean inter-arrival time, the first moment of $N_{l}^{u}$ is required.

Assume that there are $n$ time slots in the cycle. $N_{s}^{u}$ is a binomial random variable having PDF

$\operatorname{Pr}\{s$-th Tx is selected $k$ times from a total of $n$ times $\mid$ Tx- $u$ is not selected $\}=$

$\operatorname{Pr}\left\{N_{s}^{u}=k \mid n, u\right.$ is not selected $\}=\left(\begin{array}{l}n \\ k\end{array}\right)\left(\frac{\mathcal{I}_{s}}{1-\mathcal{I}_{u}}\right)^{k}\left(1-\frac{\mathcal{I}_{s}}{1-\mathcal{I}_{u}}\right)^{n-k}$ 


$$
\mathcal{I}_{u}=F_{g_{u}}\left(\frac{Q}{P}\right)\left(\prod_{i_{1}=1, i_{1} \neq u}^{L}\left(1-F_{g_{i_{1}}}\left(\frac{Q}{P}\right)\right)+\sum_{v=1}^{L-1} \sum_{j=1}^{(L-1)} \sum_{G_{0}} \operatorname{Pr}\left\{X_{j}^{v}\right\}\left(\frac{m_{h_{u}}}{\Omega_{h_{u}}}\right)^{m_{h_{u}}} \kappa_{n, p} \frac{\Gamma\left(A_{n, p}+m_{h_{u}}\right)}{\Gamma\left(m_{h_{u}}\right)}\left(B_{n}+\frac{m_{h_{u}}}{\Omega_{h_{u}}}\right)^{-\left(A_{n, p}+m_{h_{u}}\right)}\right)
$$

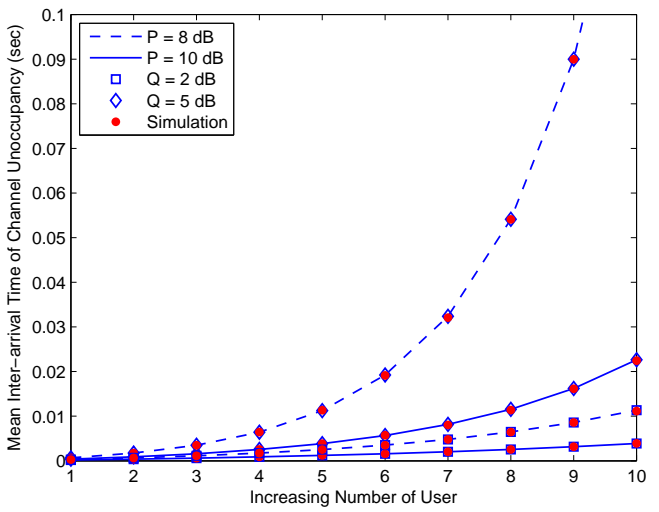

Fig. 2. Capacity of the scheduling scheme with varying peak interference power, peak transmit power and number of users, where $\Omega_{g_{l}}=$ $\{1,2,1,3,1,2,1,1,1,1\}$ and $\Omega_{h_{l}}=\{1,1,3,1,4,4,6,1,1,1\}$.

The conditional mean of $N_{s}^{u}$ is $E\left[N_{s}^{u} \mid n\right]=n\left(\frac{\mathcal{I}_{s}}{1-\mathcal{I}_{u}}\right)$, where $n$ is a geometric random variable with $\operatorname{PDF} f_{n}(n)=\mathcal{I}_{u}\left(1-\mathcal{I}_{u}\right)^{n}$. The unconditional mean of $N_{s}^{u}$ is then given by

$$
E\left[N_{s}^{u}\right]=\left(\frac{\mathcal{I}_{s}}{1-\mathcal{I}_{u}}\right) \sum_{n=0}^{\infty} n \mathcal{I}_{u}\left(1-\mathcal{I}_{u}\right)^{n}=\frac{\mathcal{I}_{s}}{\mathcal{I}_{u}} .
$$

Finally, the mean inter-arrival time of the $u$-th SU-Tx is

$$
E\left[A_{u}\right]=S \sum_{l=0, l \neq u}^{L} E\left[N_{l}^{u}\right]=S \sum_{l=0, l \neq u}^{L} \frac{\mathcal{I}_{l}}{\mathcal{I}_{u}} .
$$

The resulting mean inter-arrival time of the event that no SU$\mathrm{Tx}$ is selected for transmission can be deduced as

$$
E\left[A_{0}\right]=1-\sum_{u=1}^{L} E\left[A_{u}\right] .
$$

\section{SIMULATION RESULTS}

Numerical simulations were performed to evaluate the system performance and verify the derived results. In the simulations, $m_{h_{l}}=m_{g_{l}}=2, B=1, N_{0}=1$ and $S=1 \mathrm{~ms}$. These parameters are fixed unless stated.

Fig. 2 shows the effect on capacity when varying the peak interference power constraint. It can be observed that as the interference constraint is relaxed, the capacity of the secondary system increases. Furthermore, the capacity increases as the number of SUs increases. At low values of $Q$, increasing transmit power of the SUs results in lower capacity. This is because the number of SUs selected in the initial subset decreases. However at large $Q$, increasing transmit power increases the capacity and follows a normal trend.

Fig. 3 (Top) shows the average time after which some SU is selected and Fig. 3 (Bottom) shows the impact of number
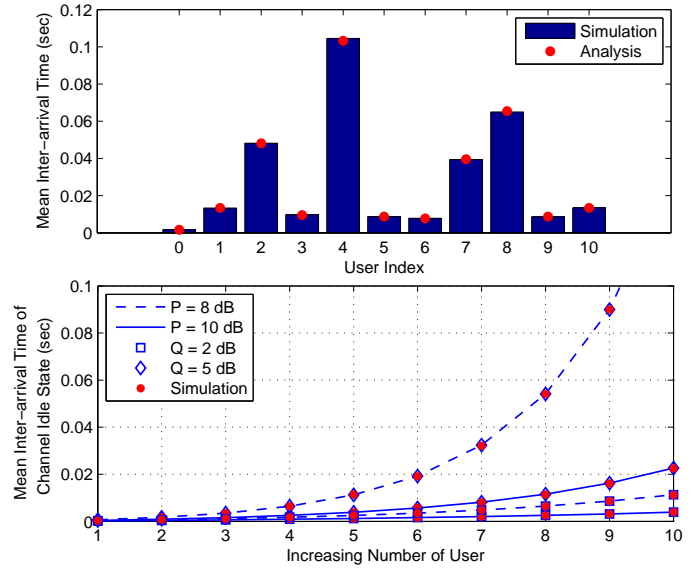

Fig. 3. Top: Mean inter-arrival time of users where $P=10 \mathrm{~dB}, Q=5 \mathrm{~dB}$, $\Omega_{g_{l}}=\{1,2,1,3,1,1,2,3,1,1\}$ and $\Omega_{h_{l}}=\{1,1,3,1,4,6,2,5,4,1\}$. Bottom: Mean inter-arrival time for channel unoccupancy with varying number of users where $m_{g_{l}}=m_{h_{l}}=1$, and $\Omega_{g_{l}}=\Omega_{h_{l}}=2, \quad \forall l$.

of SUs in the system on the inter-arrival time of channel idle state. In Fig. 3 (Top), user index '0' indicates that the channel is idle and no SU is selected. It can be observed that the average time after which a SU is selected depends on the channel statistics. A SU with a poor channel condition has a higher mean inter-arrival time of selection. In Fig. 3 (Bottom), it can be observed that as the number of SUs increase, the interarrival time of the channel idle state increases. This indicates that the channel is mostly occupied by some SU and it is very rare that some SU is not transmitting. In addition, Fig. 3 shows that as the interference power constraint is relaxed, the inter-arrival time of channel idle state increases, which is obvious. Furthermore, increasing the transmit power of the SU increases the chances that the initial subset is empty and thus, the inter-arrival time of channel idle state decreases.

\section{CONCLUSION}

We analysed the capacity and the scheduling time of users for a multiple-user underlay secondary network. The scheduling strategy consisted of two step. First, a subset of users is selected which satisfy the interference constraint and then in the second step, the user in the subset with the best secondary user forward link was selected for transmission. We obtained exact closed-from expressions for the capacity and the mean time after which a user is selected for transmission. Our results show that as the number of users increase the channel idle time (i.e. the time that no user is utilizing the channel) reduces. In addition, at low interference power, increasing the transmit power of the users is not useful and lowers the secondary network capacity. 


\section{REFERENCES}

[1] J. Mitola III and G. Maguire Jr, "Cognitive radio: Making software radios more personal," IEEE Personal Communications Magazine, vol. 6, no. 4, pp. 13-18, Aug. 1999.

[2] S. Haykin, "Cognitive radio: Brain-empowered wireless communications," vol. 23, no. 21, pp. 201-220, Feb. 2005.

[3] R. Knopp and P. Humblet, "Information capacity and power control in single-cell multiuser communications," in Proc. IEEE International Conference on Communications (ICC 1995), Seattle, WA, USA, Jun., 1995.

[4] D. Tse, "Optimal power allocation over parallel Gaussian broadcast channels," in Proc. IEEE International Symposium on Information Theory (ISIT 1997), Ulm, Germany, Jun., 1997.

[5] P. Viswanath, D. Tse, and R. Laroia, "Opportunistic beamforming using dumb antennas," IEEE Transactions on Information Theory, vol. 48, no. 6, pp. 1277-1294, Jun. 2002.

[6] M. Maso, L. Cardoso, M. Debbah, and L. Vangelista, "Channel estimation impact for MU-VFDM LTE small cells," in Proc. IEEE Global Telecommunications Conference (GLOBECOM 2011), Houston, TX, USA, Dec., 2011.

[7] S. Hua, H. Liu, M. Wu, and S. Panwar, "Exploiting MIMO antennas in cooperative cognitive radio networks," in Proc. IEEE International Conference on Computer Communications (INFOCOM 2011), Shanghai, China, Apr., 2011.

[8] T. Ban, W. Choi, B. Jung, and D. Sung, "Multi-user diversity in a spectrum sharing system," IEEE Transactions on Wireless Communications, vol. 8, no. 1, pp. 102-106, Jan. 2009.

[9] S. Ekin, F. Yilmaz, H. Celebi, K. A. Qaraqe, M.-S. Alouini, and E. Serpedin, "Capacity limits of spectrumsharing systems over hyper-fading channels," Wireless Communications and Mobile Computing, vol. 12, no. 16, pp. 1471-1480, Nov. 2012.

[10] D. Li, "On the capacity of cognitive broadcast channels with opportunistic scheduling," Wireless Communications and Mobile Computing, DOI: 10.1002/wcm.1108, 2011.

[11] R. Zhang and Y. Liang, "Investigation on multiuser diversity in spectrum sharing based cognitive radio networks," IEEE Communications Letters, vol. 14, no. 2, pp. 133-135, Feb. 2010.
[12] Y. Li and A. Nosratinia, "Hybrid opportunistic scheduling in cognitive radio networks," IEEE Transactions on Wireless Communications, vol. 11, no. 1, pp. 328 -337, Jan. 2012.

[13] I. S. Gradshteyn and I. M. Ryzhik, Table of Integrals, Series, and Products, 5th ed. Academic Press, 1994.

[14] F. Yilmaz, A. Yilmaz, M.-S. Alouini, and O. Kucur, "Transmit antenna selection based on shadowing side information," in Proc. IEEE Vehicular Technology Conference (VTC 2011 Spring), Budapest, Hungary, May, 2011.

[15] A. Annamalai, R. Palat, and J. Matyjas, "Estimating ergodic capacity of cooperative analog relaying under different adaptive source transmission techniques," in Proc. IEEE Sarnoff Symposium (Sarnoff 2010), Princeton, NJ, USA, Apr., 2010.

[16] N. Mehta, V. Sharma, and G. Bansal, "Performance analysis of a cooperative system with rateless codes and buffered relays," IEEE Transactions on Wireless Communications, vol. 10, no. 4, pp. 1069 -1081, Apr. 2011. 